\title{
Derivations of Lie Brackets and Canonical Quantisation
}

\author{
A. JOSEPH \\ Mathematical Institute, Oxford \\ Received November 13, 1969
}

\begin{abstract}
An extensive analysis of the Dirac problem of canonical quantisation is reported. In this a known solution [1] has been found to be unique to within a canonical transformation under a certain prescribed condition. This proves a conjecture due to Streater [2]. A further canonically inequivalent solution is obtained by relaxing this condition. The results obtained are discussed in terms of the derivation algebras pertaining to the Classical and Quantum Lie brackets. Applications to the study of higher symmetries and to realisations of Lie algebras as polynomial functions of canonical operators are pointed out.
\end{abstract}

\section{Introduction}

The relationship between the Poisson bracket and the commutator bracket forms the basis of one of the most important connecting links between Classical and Quantum mechanics [3]. This is because each define a Lie bracket and the corresponding Lie algebra, which may under suitable conditions be extended to a Lie group [4], then describes the underlying space-time symmetries of the mechanical system. Should we wish their space-time symmetric structures to be the same we would require these Lie algebras to be isomorphic. Such a condition leads to the important conclusion that the phase space variables which define position $q$ and canonical momentum $p$ are mapped into operators $L q$ and $L p$ satisfying the canonical commutation relations [3]. However there are certain well-known difficulties which arise in attempting to define such a transformation $[5,6]$. In particular the Poisson bracket Lie algebra of all polynomials In $q$ and $p$ is not isomorphic to the commutator bracket Lie algebra of all polynomials in $L q$ and $L p$ [6]. This important fact contributes to some of the distinguishing features of the two mechanical systems. It can give rise to ambiguities in the quantisation of Classical systems, though these can often be overcome in practice due to the simplicity of the operators involved [7]. It is pertinent to the description of the relationship between the recently discovered higher local symmetries of classical systems and their possible quantum analogues [8-10]. It results in the usual quantisation procedure not being co-ordinate free [1]. 
In the present development we give a detailed analysis of the so-called Dirac problem $[2,3]$. This is concerned with the construction of an isomorphism between the Poisson bracket Lie algebra of Classical mechanics and the commutator bracket Lie algebra of Quantum mechanics. The solution to this problem serves the purpose of defining a possible canonical quantisation procedure. Our investigation not only helps to clarify the relationship between Classical and Quantum mechanics; but at the same time we are able to propose possible alternative quantisation schemes which might prove suitable for a field theory. Thus we have proved (cf. §9) that for polynomial maps, Souriau's solution [1] is unique to within canonical equivalence. Whereas a further canonically inequivalent solution is also obtained by extending the image space of the Dirac map to include inverse powers of canonical operators.

The mathematical problems which arise in this analysis are of interest in themselves and are algebraically non-trivial. Thus we are led naturally to the problem of finding all possible realisations of a given Lie algebra by polynomial functions of canonical operators [10] and to the question of the existence of the quantum canonical conjugate of a given polynomial function $[11,12]$.

In much of the analysis to follow we consider the case of just one degree of freedom. That is we admit only one pair of canonical variables. Except in certain instances, the generalisation to an arbitrary finite number of degrees of freedom is trivial and immediate. For mathematical convenience we omit the imaginary number $i$ from the canonical commutation relations and set Planck's constant equal to one. This does not affect the analysis.

\section{Lie Brackets and Derivations}

We recall the definitions of a Lie algebra and of a derivation [13].

Definition 2.1. A Lie algebra $\mathscr{L}$ is a linear space over a field $K$ (which we shall almost always take to be the complex numbers $\mathbb{C}$ ) closed under multiplication defined by the Lie bracket [,] which satisfies:

1) $[(\alpha x+\beta y), z]=\alpha[x, z]+\beta[y, z]$,

2) $[x, y]+[y, x]=0$,

3) $[x,[y, z]]+[y,[z, x]]+[z,[x, y]]=0$,

for all $x, y, z \in \mathscr{L}$ all $\alpha, \beta \in K$. The ideal generated by all elements of the form $[x, y]: x, y \in \mathscr{L}$ is said to be the derived algebra $\mathscr{L}^{\prime}$ of $\mathscr{L}$. 
The Lie algebras with which we are concerned also possess an associative multiplication rule which satisfies

4) $[x y, z]=x[y, z]+[x, z] y$

for all $x, y, z \in \mathscr{L}$. It is the different commutativity properties of this latter multiplication which distinguishes the Classical and Quantum Lie brackets.

Definition 2.2. A derivation $D$ of a Lie algebra $\mathscr{L}$ is a linear map $D: \mathscr{L} \rightarrow \mathscr{L}$ satisfying

$$
D[x, y]=[D x, y]+[x, D y]
$$

for all $x, y \in \mathscr{L}$. A derivation $D$ is said to be inner if there exists an $x \in \mathscr{L}$ such that

$$
D y=[x, y]
$$

for all $y \in \mathscr{L}$. It is said to be outer if it is not of this form.

The set of all derivations $D(\mathscr{L})$ of $\mathscr{L}$ form a Lie algebra under the Lie bracket given by

$$
\left[D_{1}, D_{2}\right] x=D_{1}\left(D_{2} x\right)-D_{2}\left(D_{1} x\right) .
$$

This is known as the derivation algebra of $\mathscr{L}$. The set of all inner derivations $\operatorname{ad}(\mathscr{L})$ of $\mathscr{L}$ form an ideal in $D(\mathscr{L})$ homomorphic to $\mathscr{L}$. We extend the notion of derivation and inner derivation to include the following:

Definition 2.3. Given $\mathscr{L}_{1}$ a subalgebra of $\mathscr{L}_{2}$, then a derivation $D$ of $\mathscr{L}_{1}$ into $\mathscr{L}_{2}$ is a linear map $D: \mathscr{L}_{1} \rightarrow \mathscr{L}_{2}$ satisfying (2.5) for all $x, y \in \mathscr{L}_{1}$. $D$ is said to be inner if there exists an $x \in \mathscr{L}_{2}$ such that (2.6) holds for all $y \in \mathscr{L}_{1}$.

The question of the existence of outer derivations of a given Lie algebra $\mathscr{L}$ is an important one as it reflects the structure of $\mathscr{L}$. Thus if $\mathscr{L}$ is commutative, each of its inner derivations is the zero map. Whereas any non-zero linear transformation is a derivation and hence an outer derivation. At the other extreme if $\mathscr{L}$ is semisimple then all its derivations are inner (Ref. [13], p. 74). Again if the derived algebra $\mathscr{L}^{\prime}$ of $\mathscr{L}$ is semisimple and $\mathscr{L}^{\prime} \neq \mathscr{L}$ then $\mathscr{L}$ possesses both inner and outer derivations (Ref. [13], p. 103, Ex. 7). $\mathscr{L}$ also has this property if it is nilpotent (Ref. [13], p. 29, Ex. 15).

If two Lie algebras $\mathscr{L}_{1}$ and $\mathscr{L}_{2}$ are isomorphic then so are their derivation algebras $D\left(\mathscr{L}_{1}\right)$ and $D\left(\mathscr{L}_{2}\right)$. Consequently if $\mathscr{L}_{1}$ possesses outer derivations and $\mathscr{L}_{2}$ does not, then they cannot be isomorphic. 
This fact happens to be particularly useful in the comparison of the Classical and Quantum Lie brackets and leads to a better understanding of the solutions to the Dirac problem [14]. For this reason we start with some results concerning the derivations of these algebras.

\section{Characterisation Theorems for Derivations of the Poisson Bracket}

Let $P$ denote the set of all complex polynomials in the real variables $q$ and $p$. This forms an infinite dimensional Lie algebra under the Poisson bracket defined by

$$
\{f, g\}=\frac{\partial f}{\partial q} \frac{\partial g}{\partial p}-\frac{\partial f}{\partial p} \frac{\partial g}{\partial q} .
$$

In addition, (2.4) is satisfied with respect to pointwise multiplication of polynomials and this multiplication is commutative. The centre of $P$ is the one dimensional space of constant functions. Inner derivations always vanish on the centre whereas outer ones need not. The following characterisation theorem is due to Wollenberg [15].

Theorem 3.1. (Wollenberg). Every derivation $D$ of $P$ is of the form

$$
D f=\left\{a_{\alpha}, f\right\}+\beta\left(f-\alpha p \frac{\partial f}{\partial p}-(1-\alpha) q \frac{\partial f}{\partial q}\right)
$$

with $a_{\alpha} \in P$ and $\alpha, \beta \in \mathbb{C}$.

The importance of this result is that it expresses an arbitrary derivation as a sum of an inner derivation $\left\{a_{\alpha}, f\right\}$ and an explicitly determined outer derivation $\left(f-\alpha p \frac{\partial f}{\partial p}-(1-\alpha) q \frac{\partial f}{\partial q}\right)$. This decomposition is not unique and in particular the choice of $\alpha$ is arbitrary. Recalling that $D(1)=0$ for inner derivations, we see from (3.2) that $\operatorname{ad}(P)$ is an ideal of codim. 1 in $D(P)$. In the following we present a different and somewhat shorter proof than that given by Wollenberg and extend its validity to certain subalgebras of $P$. The essentially new step is contained in the following

Lemma 3.1. Given $D$ a derivation of $P$, then

$$
D f=\frac{\partial f}{\partial q} D q+\frac{\partial f}{\partial p} D p+D(1)\left(f-p \frac{\partial f}{\partial p}-q \frac{\partial f}{\partial q}\right)
$$

for all $f \in P$. 
Proof. The proof is by induction on the degree of $f$. We first note that

$$
\frac{\partial D(q)}{\partial q}+\frac{\partial D(p)}{\partial p}=\{D q, p\}+\{q, D p\}=D\{q, p\}=D(1) .
$$

Similarly it is readily verified that $D(1)$ is a constant. Expanding $\{D f, p\}$ using (2.5), (3.1) and (3.4) we obtain

$$
\{D f, p\}=D\left(\frac{\partial f}{\partial q}\right)-\frac{\partial f}{\partial q} D(1)+\frac{\partial f}{\partial p} \frac{\partial D(p)}{\partial q}+\frac{\partial f}{\partial q} \frac{\partial D(q)}{\partial q} .
$$

As $\partial f / \partial q$ is of lower degree than $f$, we may assume (3.3) to hold for $D(\partial f / \partial q)$. Then substitution of this into (3.5) gives after a little manipulation

$$
\left\{\left[D f-\frac{\partial f}{\partial q} D q-\frac{\partial f}{\partial p} D p-D(1)\left(f-p \frac{\partial f}{\partial p}-q \frac{\partial f}{\partial q}\right)\right], p\right\}=0 .
$$

A similar result may be shown to hold with $q$ replacing $p$ in the right hand position. Hence the square bracketed term is a constant $\alpha$. Setting $p=q=0$, and using the linearity of $D$ we find that $\alpha=0$. Since (3.3) holds trivially when $f$ is of degree zero, the lemma is proved.

This result gives an expression for computing $D f$ which may be compared to that for differentiation. Moreover it shows that $D$ is completely determined by its value on the canonical variables $q$ and $p$. From it the theorem follows easily. Thus for each complex number $\alpha$ we may find an $a_{\alpha} \in P$ such that

$$
\frac{\partial a_{\alpha}}{\partial p}=-(D q-\alpha q D(1)) \quad \frac{\partial a_{\alpha}}{\partial q}=(D p-(1-\alpha) p D(1)) .
$$

Indeed these equations are integrable by (3.4) and the simpleconnectedness of the Euclidean plane $E^{2}$. Substitution back into (3.3) and identification of $D(1)$ with $\beta$ gives (3.2) as required. It is in the above integration that the uniqueness of the expression for $D$ is lost.

We note in passing that Lemma 3 of Ref. [15] follows immediately from the above derivation. The theorem extends trivially to the case of $n$ degrees of freedom. It applies immediately to derivations of $P$ into $C^{\infty}$ (the space of infinitely differentiable functions). It can be extended to derivations of $C^{\infty}$ into $C^{\infty}$ by imposing suitable continuity conditions on $D$. Finally it holds for the real as well as for the complex field.

The inductive proof given in the above depends crucially on the fact that $q$ and $p$ are elements of the algebra $P$ for which we seek to construct derivations. The question then arises as to whether similar results hold 
for a subalgebra $R$ of $P$ not containing the canonical variables $q$ and $p$. As our previous considerations indicate the answer to this depends on the size of the derived algebra $R^{\prime}$. We have not attempted an exhaustive analysis of this situation, but the following result has been obtained.

Theorem 3.2. Given $R$ a subalgebra of $P$ generated by monomials with $R=R^{\prime}$. Then $\operatorname{ad}(R)$ is an ideal of codim. 0 or 1 in $D(R)$.

Proof (partial). We first identify the subalgebras $R$ of $P$ for which $R=R^{\prime}$. These are described by the following lemma.

Lemma 3.2. Let $R$ satisfy the conditions of Theorem 3.2, then one of the following hold

1) Lin. $\operatorname{span}\left(q^{2}, p^{2}, q p\right)=M_{1} \subset R$,

2) Lin. $\operatorname{span}\left(q, q p^{2}, q p\right)=M_{2} \subset R$,

3) Lin. $\operatorname{span}\left(p, q^{2} p, q p\right)=M_{3} \subset R$.

where the linear span of the said functions is taken over the complex field.

We note that the $M_{1}$ form Lie subalgebras of $R$ which are all isomorphic to $\operatorname{sl}(2, \mathbb{C})$. As this algebra is simple all the derivations of $M_{i}$ into $M_{l}$ are inner. For a proof of the theorem we must also characterise the derivations of $M_{i}$ into $R$. It turns out that these are also inner in the sense of Definition 2.3. We remark that this result depends on the particular choice of $R$ as image space of the derivations and may be shown to fail if this space is further enlarged to include inverse powers of $q$ and $p$.

We shall state without proof:

Lemma 3.3. The derivations of $M_{i}: i=1,2,3$ into $R$ are all inner.

Consider now the case $M_{1} \subset R$. The above lemma shows that given an arbitrary derivation $D$ we may subtract from it an inner derivation such that $D$ vanishes on $M_{1}$. Having done this we may show that

$$
D f=\gamma\left(q \frac{\partial f}{\partial q}+p \frac{\partial f}{\partial p}-2 f\right)
$$

for all $f \in R$ with $\gamma \in \mathbb{C}$. This defines a one parameter family of outer derivations non-zero on the complement in $R$ of $M_{1}$. Thus if $R=M_{1}$ then all the derivations of $R$ are inner. Otherwise the inner derivations form an ideal of codim.1. The argument proceeds in the same fashion for $M_{2}$ and $M_{3}$ and so the theorem is proved.

We remark that $D$ as given by (3.6) coincides with second term of (3.2) when the identifications $\alpha=1 / 2$ and $\beta=-2 \gamma$ are made. Hence essentially the same result is obtained here as before. The situation differs markedly if $R \neq R^{\prime}$. Consider for example the Lie algebra generated by the set $S=\left(q^{3}, p^{3}\right)$. Then any linear map from $S$ to $R$ defines a derivation of $R$. 
On the other hand for an inner derivation

$$
\frac{\partial}{\partial p}\left(\frac{1}{p^{2}} D\left(p^{3}\right)\right)+\frac{\partial}{\partial q}\left(\frac{1}{q^{2}} D\left(q^{3}\right)\right)=0
$$

so that the inner derivations form an ideal of infinite codim. in $D(R)$. Yet the condition that $R \neq R^{\prime}$ does not imply the existence of outer derivations. For example let $R$ be generated by the set $(q, q p)$, then $R \neq R^{\prime}$; but all the derivations of $R$ are inner. Actually this case is rather special as the Lie algebra is finite dimensional and does not involve polynomials higher than quadratic. Thus it may still be true for the infinite dimensional case that $R \neq R^{\prime}$ implies the existence of outer derivations, though we are unable to prove this conjecture.

We now turn to the corresponding problem of determining the derivations pertaining to the commutator bracket Lie algebra. Though the analysis is similar, it is yet subtly different, a circumstance which can be accredited to the noncommutativity of the associative multiplication defined in (2.4).

\section{Characterisation of Derivations for the Commutator Bracket}

Let $\mathscr{P}$ be the associative, distributive algebra over the complex field generated by finite linear combinations and finite powers of the elements $q, p$ and the identity $\mathbf{1}$, where

$$
q p-p q=\mathbf{1} .
$$

Then $\mathscr{P}$ becomes a Lie algebra with respect to the commutator Lie bracket defined by

$$
[f, g]=f g-g f
$$

for all $f, g \in \mathscr{P}$. It may be verified that the associative multiplication defined in $\mathscr{P}$ satisfies (2.4), but this multiplication is not commutative. We shall use $\mathscr{P}^{m}$ to denote the corresponding algebra generated by $m$ mutually commuting (independent) sets of elements satisfying (4.1).

We remark that no assumptions need be made at this stage concerning possible representations of $q$ and $p$. This is because the problem of determining the derivations of $\mathscr{P}$ is a purely algebraic one. For this purpose we recall some of the relevant algebraic identities. Let $a d x: x \in \mathscr{P}$ denote the linear transformation of $\mathscr{P}$ into $\mathscr{P}$ defined by

$$
a d x: y \rightarrow(a d x) y=[x, y]
$$

for all $y \in \mathscr{P}$. Then by induction on the recurrence relation

$$
a d x^{m+1}=x a d x^{m}-a d x a d x^{m}+x^{m} a d x
$$


it may be shown that

$$
a d x^{m}=\sum_{k=1}^{m}\left(\begin{array}{l}
m \\
k
\end{array}\right)(-1)^{k-1} x^{m-k} a d^{k} x .
$$

This holds for all positive integer $m$. As a special case setting $x=p$ we obtain

$$
\left[q, p^{n}\right]=n p^{n-1}
$$

for all positive integer $n$. Combining (4.3) and (4.4) then gives

$$
\left[q^{m}, p^{n}\right]=\sum_{k=1}^{(m, n)}\left(\begin{array}{l}
m \\
k
\end{array}\right)\left(\begin{array}{l}
n \\
k
\end{array}\right)(-1)^{k-1} k ! q^{m-k} p^{n-k}
$$

where $(m, n)$ denotes the smaller of the two integers $m$ and $n$. (Eq. (4.5) has been given by Rosenbaum [16] whose derivation we have simplified.)

From (4.4) it may be seen that $a d q$ acts like differentiation on polynomials of $p$. A similar statement applies to $a d p$. A particular consequence of this fact is that if an element $x \in \mathscr{P}$ commutes with $q$ (or $p$ ) then it is independent of $p$ (or $q$ ). Hence the centre of $\mathscr{P}$ is the one dimensional space of constant multiples of $\mathbf{1}$. These facts play an important role in the subsequent analysis as they do also in the representation theory (Ref. [7], pp. 137-138).

We are now ready to present the main result of this section.

Theorem 4.1. The derivations of $\mathscr{P}$ are all inner.

Proof (partial). There are two stages in the proof. We first show:

Lemma 4.1. A derivation $D$ of $\mathscr{P}$ is inner if and only if $D(\mathbf{1})=0$.

Proof. Necessity is immediate because an inner derivation must vanish on the centre. The proof of sufficiency is by induction on the degree of $f$.

We next show that for any derivation $D$ of $\mathscr{P}, D(\mathbf{1})=0$. To this end we note without proof:

Lemma 4.2. Given $D$ a derivation of $\mathscr{P}$ for which $D(\mathbf{1})=\alpha \mathbf{1}: \alpha \in \mathbb{C}$, then modulo an inner derivation

$$
D\left(q^{m} p^{n}+p^{n} q^{m}\right)=\frac{\alpha}{2}(2-m-n)\left(q^{m} p^{n}+p^{n} q^{m}\right)
$$

for all positive integers $m$ and $n$.

The theorem now follows easily. From (4.6) we obtain

$$
D\left[q^{m}, p^{n}\right]=\left[D\left(q^{m}\right), p^{n}\right]+\left[q^{m}, D\left(p^{n}\right)\right]=\frac{\alpha}{2}(4-m-n)\left[q^{m}, p^{n}\right] .
$$


Recombined with (4.6) we have by the linearity of $D$ that

$$
D\left(q^{m} p^{n}\right)=\frac{\alpha}{2}(2-m-n) q^{m} p^{n}+\frac{\alpha}{2}\left[q^{m}, p^{n}\right] .
$$

From this may recompute $D\left[\mathrm{q}^{m}, p^{n}\right]$ using (4.5). To do so we require the identity

$$
\begin{aligned}
\sum_{k=1}^{(m, n)} \sum_{t=1}^{(m-k, n-k)}(-1)^{k+t} & \frac{m ! n !}{(m-k-t) !(n-k-t) ! k ! t !} q^{m-k-t} p^{n-k-t} \\
& =\sum_{u=1}^{(m, n)}(-1)^{u} \frac{m ! n !}{(m-u) !(n-u) ! u !} q^{m-u} p^{n-u} .
\end{aligned}
$$

This may be verified by making the change of variable defined by $2 k=u+v, 2 t=u-v$ and summing over $v$. Then comparing (4.7) with the expression for $D\left[\mathrm{q}^{m}, p^{n}\right]$ which results on first expanding the argument $\left[q^{m}, p^{n}\right]$ using (4.5) and substituting from (4.8) and (4.9), we obtain

$$
\alpha \sum_{k=1}^{(m, n)}(-1)^{k}\left(\begin{array}{l}
m \\
k
\end{array}\right)\left(\begin{array}{l}
n \\
k
\end{array}\right)\left(2^{k}-2 k\right) k ! q^{m-k} p^{n-k}=0 .
$$

The term inside the summation cannot be zero if both $m, n \geqq 3$. Thus $\alpha=0$ and the theorem is proved.

Though $\mathscr{P}$ itself does not admit outer derivations, there are a number of important subalgebras of $\mathscr{P}$ which do admit them. Let us denote by $\mathscr{R}_{1}, \mathscr{R}_{2}, \mathscr{R}_{3}$ the subalgebras of $\mathscr{P}$ defined as the linear span of the sets $S_{1}, S_{2}, S_{3}$ where

$$
\begin{aligned}
& S_{1}=\left(\mathbf{1}, q, p, q^{2}, p^{2}, q p\right), \\
& S_{2}=\left(\mathbf{1}, q^{m+1}, q^{m} p: m \text { a non-negative integer }\right), \\
& S_{3}=\left(\mathbf{1}, q^{m} p^{n}+p^{n} q^{m}: m, n \text { positive integers }\right) .
\end{aligned}
$$

In addition we shall use $\mathscr{R}_{i}{ }^{m}: i=1,2,3$ to denote the corresponding algebras generated by $m$ independent sets of canonical operators. Then from the proof of the above theorem we have the immediate corollary.

Corollary 4.1. The inner derivations of $\mathscr{R}_{i}{ }^{m}: i=1,2,3 ; m$ a finite positive integer, form an ideal of codim. 1 in $D\left(\mathscr{R}_{i}{ }^{m}\right)$. For $\mathscr{R}_{1}$ and $\mathscr{R}_{2}$ the outer derivations are given by (4.8) with $n=1,2$. For $\mathscr{R}_{3}$ the outer derivations are given by (4.6).

It turns out [17] and Ref. [7], p. 141, that $\mathscr{R}_{1}$ and $\mathscr{R}_{2}$ are just the subalgebras of $\mathscr{P}$ which admit an isomorphism with the corresponding subalgebras defined with respect to the Poisson bracket. This circumstance indicates that we may anticipate less difficulty in the quantisation of simple mechanical systems. Thus the Hamiltonian for the harmonic 
oscillator and the Runge-Lenz vector of the Coulomb problem are readily translated to quantum mechanical form. In addition $\mathscr{R}_{2}$ turns out to be particularly useful in the understanding of the solutions to the Dirac problem. The example of $\mathscr{R}_{3}$ for its part suggests that we might avoid the difficulties arising in quantisation by symmetrisation of the polynomials concerned. However this procedure cannot be extended consistently to the whole algebra, though in effect the quantisation of $\mathscr{R}_{1}$ and $\mathscr{R}_{2}$ take this form.

\section{The Dirac Problem}

Let $\mathscr{L}(\mathscr{H})$ denote the linear (but not necessarily bounded or selfadjoint) operators acting on some Hilbert space $\mathscr{H}$, then

Definition 5.1. A Dirac map [2] is a linear map $L$ from $P$ into $\mathscr{L}(\mathscr{H})$ such that $L(1)=\mathbf{1}$ and

$$
[L f, L g]=L\{f, g\}
$$

for all $f, g \in P$.

Such a map defines an isomorphism between the Lie algebras formed from the Poisson and commutator brackets. Its importance lies in the fact that it provides a possible canonical quantisation procedure for Classical systems. The Dirac problem is to find all such Dirac maps. One important class can be obtained as follows $[2,15]$. Denote by $C_{0}^{\infty}\left(E^{2}\right)$ the space of infinitely differentiable functions with compact support in $E^{2}$ and let $\mathscr{H}$ be $L^{2}\left(E^{2}\right)$, the space of square integrable functions on $E^{2}$. Let $D$ be an outer derivation of $P$ into $C^{\infty}\left(E^{2}\right)$ with $D(1)=\mathbf{1}$. Then the map $f \rightarrow L f$ given by

$$
(L f) \phi=\{f, \phi\}+(D f) \phi
$$

with $\phi \in C_{0}^{\infty}\left(E^{2}\right)$ is a Dirac map. ( $L f$ is well-defined because $(L f) \phi$ is square integrable and defined on a dense subspace of $\mathscr{H}$ because $C_{0}^{\infty}\left(E^{2}\right)$ is dense in $L^{2}\left(E^{2}\right)$.) In particular if we choose

$$
D f=f-\frac{1}{2} q \frac{\partial f}{\partial q}-\frac{1}{2} p \frac{\partial f}{\partial p}
$$

then $L f$ takes the explicit form

$$
L f=\frac{\partial f}{\partial q} \frac{\partial}{\partial p}-\frac{\partial f}{\partial p} \frac{\partial}{\partial q}+f-\frac{1}{2} q \frac{\partial f}{\partial q}-\frac{1}{2} p \frac{\partial f}{\partial p} .
$$

There is in fact no loss in generality in doing this as the more general expression given in (5.2) can be reduced to (5.4) by a canonical transformation (Ref. [2], pp. 364-366). 
Inspection of (5.4) shows that the expression for $L f$ involves two independent sets of quantum canonical operators. That is to say that the image of $P$ under $L$ is not $\mathscr{P}$ as might be hoped; but rather a subalgebra of $\mathscr{P}^{2}$. This subalgebra is in fact $\mathscr{R}_{2}{ }^{2}$ which was defined in the previous section. Thus the present solution of the Dirac problem does not provide an isomorphism of $P$ with $\mathscr{P}$, but rather an isomorphism of $P$ with $\mathscr{R}_{2}{ }^{2}$. This result is in accord with our previous study of their derivation algebras. Nevertheless this situation may be thought to be rather unsatisfactory as the implied quantisation procedure does not correspond to the one customarily used. For certain simple mechanical problems, in particular the harmonic oscillator, this difficulty is less important because the quantum mechanical Hamiltonian derived by the above procedure can be decomposed into two copies of that usually obtained (Ref. [2], pp. 359-361). This observation is particularly relevant to field theory because of the importance of this Hamiltonian in the description of the free field and because the infinite number of degrees of freedom makes this doubling irrelevant. It is a situation which can be more directly investigated through the notion of an irreducible Dirac map. This we do in the following section. In later sections we investigate the general Dirac problem and in particular show that the solution described by (5.4) is canonically equivalent to all other solutions in $\mathscr{P}^{2}$. We also obtain a further canonically inequivalent solution by extending the image space to include inverse powers of the canonical operators. Finally we discuss possible applications of these results and related work.

\section{Irreducible Dirac Maps}

Definition 6.1. A Dirac map $L$ of $P$ into $\mathscr{L}(\mathscr{H})$ is said to be irreducible if the only operators commuting with both Lq and Lp are constant multiples of the identity.

Recalling Definition 5.1 we see that

$$
[L q, L p]=\mathbf{1} \text {. }
$$

Thus $L q$ and $L p$ are quantum canonical operators and an irreducible Dirac map defines an isomorphism between $P$ and a subalgebra of $\mathscr{P}$. Certainly $P$ and $\mathscr{P}$ are not isomorphic as one possesses outer derivations whilst the other does not. Therefore it is not surprising that one can show that there are no irreducible maps. Though this result is a negative one, we are able at the same time to determine those subalgebras of $P$ which do admit irreducible Dirac maps and to prepare the discussion for the general Dirac problem. 
Theorem 6.1. There are no irreducible Dirac maps of $P$ into $\mathscr{L}(\mathscr{H})$.

Proof (partial). The proof is by contradiction. We note without proof that:

Lemma 6.1. Given $L$ an irreducible Dirac map, then

$$
L\left(q^{m} p^{n}\right)=\frac{1}{(m+1)(n+1)}\left[L^{m+1}(q), L^{n+1}(p)\right]
$$

for all positive integers $m$ and $n$.

On the other hand we may also show

Lemma 6.2. Given $L$ an irreducible Dirac map, then

$$
L\left(q^{m} p^{n}\right)=L^{m}(q) L^{n}(p)+L^{n}(p) L^{m}(q)
$$

for all positive integer $m$ and $n$.

These lemmas lead to a contradiction for $m, n \geqq 3$ and so the theorem is proved.

Corollary 6.1. Let $R_{1}$ and $R_{2}$ denote the subalgebras of $P$ generated by the sets $S_{1}$ and $S_{2}$ defined in (4.10), then there exist irreducible Dirac maps $R_{1}$ and $R_{2}$ into $\mathscr{L}(\mathscr{H})$. In both cases the map is given by (6.3).

A corresponding result does not hold for $R_{3}$, the subalgebra of $P$ generated by $S_{3}$, because this coincides with $P$.

\section{Finite Dimensional Dirac Maps}

Consider the subalgebra $R_{1}$ of $P$. A simple calculation shows that

$$
\begin{aligned}
L\left(q^{2}\right) & =L^{2}(q)+2 f, \\
L\left(p^{2}\right) & =L^{2}(p)+2 g, \\
L(q p)=L(p q) & =\frac{1}{2}(L q L p+L p L q)+2 h
\end{aligned}
$$

where $f, g, h \in \mathscr{L}(\mathscr{H})$, commute with $L q$ and $L p$ and satisfy

$$
[f, g]=2 h, \quad[f, h]=f, \quad[g, h]=-g .
$$

This last set of relations define the Lie algebra of $\operatorname{sl}(2, \mathbb{C})$. Hence the solution to the Dirac problem for $R_{1}$ is provided by the representations of this algebra. As these are well understood we may regard the Dirac problem to be completely solved for this case. We should like to extend this argument to the general Dirac problem. However it turns out that this is several orders of magnitude more difficult. Therefore we first investigate the simpler problem motivated by the following consideration. 
It is clear that (7.4) has finite dimensional representations. In particular we may set $f, g, h$ equal to a suitable linear combination of the Pauli spin matrices. Such an occurrence of finite dimensional representations, as a result of a canonical quantisation procedure, would be of considerable physical interest. Hence the question arises as to whether finite dimensional representations of (7.4) suffice to describe a Dirac map of $P$. It is unfortunate and perhaps not surprising, taking into account that canonical operators may be involved, that the answer is negative. Nevertheless the proof is of interest in itself and is non-trivial. Moreover many of the relations we obtain are required in the solution of the general Dirac problem. To make our objectives more precise we start with the following definition.

Definition 7.1. A Dirac map $L$ of $P$ into $\mathscr{L}(\mathscr{H})$ is said to be finite dimensional of dimension $m$ if every operator which commutes with both $L q$ and $L p$ is a tensor product of the identity and an $(m \times m)$ matrix.

In the above terminology the irreducible Dirac maps are one dimensional. By a slight abuse of notation we omit further explicit reference to the tensor product. However this should cause no confusion.

The main result of this section can be stated as follows:

Theorem 7.1. There are no finite dimensional Dirac maps of $P$ into $\mathscr{L}(\mathscr{H})$.

Proof (partial). We first note some important algebraic identities. The first of these is described in the following lemma.

Lemma 7.1. Given a Dirac map $L$ of $P$ into $\mathscr{L}(\mathscr{H})$, then

$$
\begin{aligned}
& L\left(q^{m}\right)=\sum_{k=0}^{m}\left(\begin{array}{l}
m \\
k
\end{array}\right) a_{m-k} L^{k}(q), \\
& L\left(p^{m}\right)=\sum_{k=0}^{m}\left(\begin{array}{l}
m \\
k
\end{array}\right) b_{m-k} L^{k}(p)
\end{aligned}
$$

for all positive integer $m$, where $a_{k}, b_{k} \in \mathscr{L}(\mathscr{H}): k=0,1,2 \ldots m$ and commute with both $L q$ and $L p$. In addition

$$
a_{0}=b_{0}=1, \quad a_{1}=b_{1}=0
$$

Lemma 7.2. The $a_{k}: k=0,1,2 \ldots$ defined in the previous lemma satisfy the following identities

and

$$
\left[a_{k}, a_{l}\right]=0
$$

$$
\left[a_{k}, h\right]=\frac{k}{2} a_{k}
$$

for all non-negative integer $k$ and $l ; h$ being defined in (7.3). 
As an immediate consequence of the previous lemma and (7.4) we obtain the following identities

$$
\left[\left(a d^{k} g\right) a_{m}, h\right]=(m / 2-k)\left(a d^{k} g\right) a_{m}
$$

for all positive integer $m$ and $k$ and

$$
\left(a d^{l} f\right)\left(a d^{k} g\right) a_{m}=(-1)^{l} \frac{(m-k+l) ! k !}{(k-l) !(m-k) !}\left(a d^{k-l} g\right) a_{m}
$$

for all integer $m, k, l$ with $m \geqq k \geqq l$. From (7.10) and (7.11) it can be seen that if $a_{m} \neq 0$ then the operators $\left(a d^{k} g\right) a_{m}: k=0,1,2 \ldots m$ are non-zero and linearly independent. As the $(m \times m)$ matrices form a space of dimension $\mathrm{m}^{2}$, we obtain from this the following result:

Lemma 7.3. If $L$ is an m-dimensional Dirac map, then $a_{m^{2}}=0$.

The proof is completed by showing that this leads to a contradiction.

\section{The Operators $a_{k}$}

Recalling (5.1) we see from (7.5) and (7.6) that the Dirac problem is completely solved when we have the most general expressions for the $a_{k}$ and the $b_{k}$. Now these are not all independent. In the first place the $b_{k}: k \geqq 3$, can be obtained from the $a_{k}$ by $k$-fold application of the linear transformation $a d g$. This follows from the identity

$$
\left(a d^{k} L\left(p^{2}\right)\right) L\left(q^{k}\right)=(-2)^{k} k ! L\left(p^{k}\right)
$$

which gives

$$
b_{k}=(-1)^{k}\left(a d^{k} g\right) a_{k} .
$$

Secondly the $a_{k}$ are themselves interdependent. We have already found (cf. (7.8)) that they commute. We show below that they are determined by $a_{2}$ and $a_{3}$ alone. This fact is contained in the following lemma.

Lemma 8.1. Given the $a_{k}$ as defined in Lemma 7.1, then

$$
a_{m+n}=a_{m} a_{n}+\frac{1}{(m+1)(n+1)}\left[a_{m+1},\left[a_{n+1}, g\right]\right]
$$

for all non-negative integer $m$ and $n$.

Recalling (7.7) we see from this that the general term $a_{m}$ is determined by $a_{2}, a_{3}$ and $g$. In addition, $a_{2}$ and $a_{3}$ cannot be wholly independent as (8.2) implies certain consistency relations between them. Unfortunately it is not easy to resolve the precise form of this interdependence, a fact which is in turn responsible for much of the difficulty that arises in the Dirac problem. It can be overcome in the case of polynomial Dirac maps 
as we show in the next section. For the present we investigate the solution prescribed by the condition

$$
a_{3}=0 .
$$

In this case (8.2) simplifies considerably giving

$$
a_{2 n+1}=0, \quad a_{2 n}=a_{2}{ }^{n}
$$

for all non-negative integer $n$. The main result of this section can then be stated as follows:

Theorem 8.1. There are no Dirac maps satisfying (8.3).

The importance of this result is that the condition $a_{3} \neq 0$ puts an additional constraint on the possible solutions of (7.4). Indeed, setting $k=3$ in (7.9), we see that half-integer as well as integer steps in the spectrum of $h$ (in that it has a spectrum) can be effected. As we shall see in the next section certain solutions of (7.4) are thereby excluded and this leads ultimately to a complete solution of the polynomial Dirac problem.

\section{Polynomial Solutions to the Dirac Problem}

In the following we derive the solutions to the Dirac problem in the case when $f, g, h$ and $a_{3}$ are polynomials in a pair of canonical operators. In the terminology of Definition 7.1 such solutions are infinite dimensional so that the present analysis to some extent complements that given in $\S 7$. The advantage of studying the polynomial solutions is that we may put aside questions of selfadjointness, boundedness and domains of the operators concerned and treat the problem on a purely algebraic level. This is particularly useful in the present situation as the solutions to the Dirac problem are likely to involve operators which satisfy the canonical commutation relations. Such operators are unbounded, have continuous spectra and incomplete domains and consequently are less easy to handle on a functional analytic level [18].

To clarify our objectives we start with the definition.

Definition 9.1. A Dirac map $L$ of $P$ into $\mathscr{L}(\mathscr{H})$ is said to be a polynomial map of degree $n$ if for each $f \in P, L f$ is a polynomial in not more than $n$ independent sets of canonical operators.

In the above terminology the irreducible Dirac maps are polynomial maps of degree one. We are able to show that to within a canonical transformation the Dirac map given by (5.4) exhausts all possible polynomial maps of degree two. On the other hand we also exhibit a further canonically inequivalent solution involving inverse powers of canonical operators. 
The simplification which comes from restricting to polynomial maps of degree two is a consequence of the following theorem.

Theorem 9.1. Given a, $f, h \in \mathscr{P}$ with

$$
[a, f]=0, \quad[f, h]=f, \quad[a, h]=3 a / 2
$$

then there exist constants $\alpha, \beta$ such that

$$
\alpha a^{2}+\beta f^{3}=0 .
$$

Proof (partial). The key step in the proof comes from the lemma.

Lemma 9.1. Given $a, b \in \mathscr{P}$ with $[a, b]=0$. Let $\mathscr{R}$ be the set of all polynomials in $a, b$ ordered so that powers of a appear to the left. Then there exists a non-trivial element of $\mathscr{R}$ which is independent of $q$ and $p$.

Applied to (9.1a) this lemma shows that there exists an ordered polynomial of $a, f$ and $\mathbf{1}$ which vanishes. We may then use $(9.1 \mathrm{~b}, \mathrm{c})$ to show that this must be a polynomial with factors of the form given in the lefthand side of (9.2). At least one of these factors must vanish identically and so the theorem is proved.

The simplification arising from (9.2) is considerable. Applied to the Dirac problem setting $a=a_{3}$ we are able to assert that $f$ is a constant multiple of the square of a canonical operator. This in turn leads to the following characterisation theorem for polynomial Dirac maps.

Theorem 9.2. Given $L$ a polynomial Dirac map of degree two with $q_{i}, p_{j}: i, j=1,2$ satisfying

$$
\left[q_{i}, p_{j}\right]=\delta_{i j} 1
$$

where $\delta_{i j}$ is the Kronecker delta, then to within a canonical transformation

$$
L f=\left(\frac{\partial f}{\partial q}\right)^{*} q_{2}-\left(\frac{\partial f}{\partial p}\right)^{*} q_{1}+f^{*}-\frac{1}{2} p_{1}\left(\frac{\partial f}{\partial q}\right)^{*}-\frac{1}{2} p_{2}\left(\frac{\partial f}{\partial p}\right)^{*}
$$

for all $f \in P$. Here * denotes that the real variables $q$, $p$ have been replaced by the commuting operators $p_{1}, p_{2}$ in the given function.

Proof (partial). One pair of canonical operators, namely: $L q$ and $L p$ is provided by (6.1). Moreover these commute with the $a_{k}, b_{k}: k=1,2 \ldots$ defined $\S 7$. Thus the problem of determining all polynomial solutions of degree two reduces to finding the most general realisation of the $a_{k}$ and the $b_{k}$ as polynomials in a single pair of canonical operators. Thus applying Theorem 9.1 to the identities given in (7.8), (7.9) and (8.2) and substituting in (7.5) and (7.6) we may show that to within a canonical 
transformation

$$
\begin{aligned}
& L\left(q^{m}\right)=\sum_{k=0}^{m}(m-k-1)(-1)^{m-k-1}\left(\begin{array}{c}
m \\
k
\end{array}\right) x^{m-k} L^{k}(q), \\
& L\left(p^{n}\right)=\sum_{l=0}^{n}(n-l-1)(-1)\left(\begin{array}{l}
n \\
l
\end{array}\right) y^{n-l} L^{l}(p)
\end{aligned}
$$

where $x, y$ satisfy

$$
[x, y]=\mathbf{1} .
$$

Applying the canonical transformation

$$
\begin{array}{ll}
L q=q_{2}+\frac{1}{2} p_{1} \quad x=q_{2}-\frac{1}{2} p_{1} \\
L p=-q_{1}+\frac{1}{2} p_{2} \quad y=q_{1}+\frac{1}{2} p_{2}
\end{array}
$$

to (9.4) and (9.5) we obtain (9.3) as required, and the theorem is proved.

We remark in passing that the Dirac map defined above is based on the following realisation of $\operatorname{sl}(2, \mathbb{C})$ in $\mathscr{P}$, namely

$$
f=\frac{-x^{2}}{2}, \quad g=\frac{-y^{2}}{2}, \quad h=\frac{1}{4}(x y+y x) .
$$

This is not the only possible realisation; but it is the only consistent with $a \neq 0$ as required by Theorem 8.1 . Thus we may set [19]

$$
f=\frac{1}{2}\left(x^{2} y+y x^{2}\right), \quad g=y, \quad h=\frac{1}{2}(x y+y x),
$$

and it may be verified that this is also consistent with (7.4). On the other hand in this case neither $f$ of $g$ admit a square root in $\mathscr{L}(\mathscr{H})$ and this excludes the above realisation when $a \neq 0$.

\section{A Solution to the Dirac Problem in Inverse Powers of Canonical Operators}

In the previous section we noted that a known solution of the polynomial Dirac problem was unique to within canonical equivalence. Though this is a non-trivial result in its own right, it cannot of itself give rise to any interesting new solutions. Yet, as a by-product of this analysis, we are able to obtain an additional solution canonically inequivalent to (9.3). This involves inverse powers of the canonical operators $q, p$ which we define in the usual way, namely

$$
q q^{-1}=q^{-1} q=\mathbf{1}, \quad p p^{-1}=p^{-1} p=\mathbf{1} .
$$


We denote by 2 the polynomial algebra generated by $q, p, q^{-1}, p^{-1}$. The general commutator in 2 may be obtained from (4.5) and the relation

$$
\left[q^{-m}, p^{-n}\right]=\sum_{k=1}^{m}(-1)^{k-1} k !\left(\begin{array}{l}
m \\
k
\end{array}\right) \frac{\Gamma(n+k)}{\Gamma(n) \Gamma(k+1)} q^{-1} q^{-(m-k)} p^{-(n+k)} q^{-1}
$$

which holds for all positive integer $m$ and integer $n$. This may be developed by the procedure described in $\S 4$. A similar expression has been given by Rosenbaum [16].

We shall extend the argument of the previous section to 2 . In this we do not need to consider possible representations of these operators or their analytic properties. This is because the mathematical argument, and a rigorous one at that, can proceed at purely algebraic level. However for the sake of further application we mention a few of their more important properties.

Suppose we choose $q$ and ip to be self-adjoint elements of $\mathscr{L}(\mathscr{H})$, $i=\sqrt{-1}$. Then it may be shown that (4.1) implies that they each have a continuous spectrum which consists of the whole of the real line [18]. Hence their inverses $q^{-1}$ and $(i p)^{-1}$ also have this property. Let $\mathscr{L}^{2}(-\infty, \infty)$ be the space of square integrable functions on the real line with $x$ a real variable. Then in $\mathscr{L}\left(\mathscr{L}^{2}(-\infty, \infty)\right) q$ and $p$ have representations as multiplication by $x$ and as differentiation with respect to $x$ [18]. Correspondingly $q^{-1}$ and $p^{-1}$ may be represented by multiplication by $x^{-1}$ and by the integral operator with kernel $h\left(x-x^{\prime}\right)$ where $h$ is Heaviside step function. Perhaps the most important physical system in which such inverse powers occur is the hydrogen atom. In this the coulombic term is represented as multiplication by $r^{-1}$ in position space and by the integral operator with kernel $\left|\boldsymbol{p}-\boldsymbol{p}^{\prime}\right|^{-2}$ in momentum space $[20]$.

We do not attempt to give a complete account of Dirac maps in 2 , as we have yet to prove an analogue of Theorem 9.1 for this case. Also we feel that 2 may be an unsuitable object for a general analysis. Thus it is not even closed under linear canonical transformations so that statements about 2 are not canonically invariant. Whilst this difficulty could be corrected, it is better left as a subject for a later discussion.

We are now able to state the main result of this section.

Theorem 10.1. The linear map $L: \mathscr{L}(\mathscr{H})$ given by

$$
\begin{aligned}
& L\left(q^{m}\right)=\sum_{k=0}^{m}(m-k-1)(-1)^{m-k-1}\left(\begin{array}{l}
m \\
k
\end{array}\right) x^{m-k} L^{k}(q), \\
& L\left(p^{n}\right)=\sum_{l=0}^{n}(n-l-1)(-1)\left(\begin{array}{l}
n \\
l
\end{array}\right)\left(y-(x y x)^{-1}\right)^{n-l} L^{l}(p),
\end{aligned}
$$

16 Commun math. Phys., Vol 17 
(6.1) and (9.6) define a Dirac map. Furthermore this map is not canonically equivalent to that given in (9.3).

The above solution to the Dirac problem is based on some rather remarkable identities involving canonical operators. First of all the expressions for $f, g$ and $h$ which define a realisation of $s l(2, \mathbb{C})$ take the form

$$
f=-\frac{x^{2}}{2}, \quad g=-\frac{1}{2}\left(y^{2}-2 x^{-2}\right), \quad h=\frac{1}{4}(x y+y x)
$$

where $x, y$ satisfy (9.6). This solution is not canonically equivalent to that given in (9.8). Furthermore the asymmetry in $f$ and $g$ which leads to a corresponding asymmetry in $L\left(q^{m}\right)$ and $L\left(p^{n}\right)$ is only apparent. To see this we first note the surprising fact that $g$ has a square root in 2 . This is contained in the identity

$$
\hat{y}^{m} \equiv\left(y-(x y x)^{-1}\right)^{m}=y^{m}-m x^{-1} y^{m-2} x^{-1}
$$

which holds for all integer $m$. In addition to this, $\hat{y}$ is canonically conjugated in 2 . That is there exists an element $\hat{x} \in \mathscr{Q}$ for which

$$
[\hat{x}, \hat{y}]=1 \text {. }
$$

Indeed $\hat{x}$ is given by

$$
\hat{x}=x+\frac{1}{2}\left(y^{-2} x^{-1}+x^{-1} y^{-2}\right)
$$

as may be verified using (10.2). Inversion formulae which express $x$ and $y$ in terms of $\hat{x}$ and $\hat{y}$ are also available and these take the form

$$
\begin{aligned}
& x=\hat{x}-(\hat{y} \hat{x} \hat{y})^{-1}, \\
& y=\hat{y}+\frac{1}{2}\left(\hat{x}^{-2} \hat{y}^{-1}+\hat{y}^{-1} \hat{x}^{-2}\right) .
\end{aligned}
$$

Substitution of these expressions into (10.4) interchanges the roles of $f$ and $g$ and this in effect restores the symmetry to this realisation of $\operatorname{sl}(2, \mathbb{C})$.

Finally we remark that the canonical transformation (9.7) applied to $(10.3 \mathrm{a}, \mathrm{b})$ ultimately leads to the following expression for $L$ on a general monomial. This is

$$
\begin{aligned}
L\left(q^{m} p^{n}\right)= & m p_{1}^{m-1} p_{2}^{n} q_{2}-n p_{1}^{m} p_{2}^{n-1} q_{1}+\frac{1}{2}(2-m-n) p_{1}^{m} p_{2}^{n} \\
& +n(n-1) p_{1}^{m}\left(q_{2}-\frac{1}{2} p_{1}\right)^{-1} p_{2}^{n-2}\left(q_{2}-\frac{1}{2} p_{1}\right)^{-1}:
\end{aligned}
$$

This differs from the expression obtained from (9.3) in the presence of the last term. 


\section{Canonical Conjugation and Higher Symmetries}

In $\S 5$ we observed that $P$ and $\mathscr{P}$ are not isomorphic. This fact is responsible for a number of differences between Classical and Quantum mechanics. Perhaps the most striking of these occurs in the problem of canonical conjugation. This is the problem of determining the canonical conjugate of a given function in $q$ and $p$. The situation in Classical mechanics is well understood and we shall examine this first $[4,9,21]$.

Let $A$ denote the algebra of all complex analytic functions in the real variables $q$ and $p$. Then given any element $x \in A$, there exists an element $y \in A$ such that

$$
\{x, y\}=1 \text {. }
$$

To show this we solve the differential equations

$$
\frac{d q}{d y}=-\frac{\partial x}{\partial p}, \quad \frac{d p}{d y}=\frac{\partial x}{\partial q}
$$

which have analytic solutions because $x$ is analytic. We then invert these expressions to obtain $y$ as an analytic function of $q$ and $p$. At the same time we obtain

$$
\frac{d}{d y}=\frac{\partial x}{\partial q} \frac{\partial}{\partial p}-\frac{\partial x}{\partial p} \frac{\partial}{\partial q} \text {. }
$$

This implies (11.1) and so $y$ is the required analytic function.

This physical interpretation of the above transformation comes from the identification of $x$ with the Hamiltonian. Then (11.2) become Hamilton's equations of motion and the $y$-dependence of $q$ and $p$, the path in phase space which describes the time evolution of the mechanical system [21].

In Quantum mechanics the analysis is less straightforward and it is not known if a given function of the canonical operators $q$ and $p$ can be canonically conjugated in any well-defined sense. Indeed the functional analytic approach would seem to indicate that this will not be the case in general. This is because, as we have already noted, the operators which satisfy (4.1) must have each a rather special type of spectrum. Hence an operator not having such a spectrum ${ }^{1}$ cannot be canonically conjugated in $\mathscr{L}(\mathscr{H})$. The corresponding physical interpretation [11] of this is that we cannot express Quantum mechanical time as an element of $\mathscr{L}(\mathscr{H})$. This does not exclude the possibility of being able to develop a formal power series [12] for the time, because such an expression may not converge (in any sense) to an element of $\mathscr{L}(\mathscr{H})$. Without this convergence the operators lose their usual meaning and so we feel should not be taken into consideration here.

\footnotetext{
${ }^{1}$ For example the harmonic oscillator Hamiltonian which has a discrete positive spectrum.
} 
There is of course one important exception to the above. This is that any linear function in $q$ and $p$ can be canonically conjugated in $\mathscr{P}$. The canonical transformations so defined are said to be sympletic and these play an important role in Quantum symmetries. On the other hand the canonical conjugation of elements of $\mathscr{P}$ of higher degree is restricted by the fact that the commutator does not generally lower the combined degree by more than two. The same holds true of the Poisson bracket and this is why we chose to discuss the algebra $A$ rather than $P$. However unlike the Classical case, there is no obvious extension of $\mathscr{P}$ in $\mathscr{L}(\mathscr{H})$ which admits canonical conjugation of its elements.

Recalling (10.5)-(10.8) we see that 2 may admit canonical transformations not available to $\mathscr{P}$. Actually as far as we know this is the first example of its kind obtained within the framework of Hilbert space for which the corresponding functions in the real variables $q$ and $p$ are not canonical conjugates under the Poisson bracket. It serves to extend the class of non-linear functions which can admit canonical conjugation. It would be of interest to complete this list.

Thus far we have only considered the case of one degree of freedom. When there are $n$ degrees of freedom we may find $[9,22]$, for a given element $x_{1} \in A^{n},(2 n-1)$ further elements, $x_{l}: i=2,3 \ldots n ; y_{j}: j=1,2 \ldots n$; $x_{i}, y_{j} \in A^{n}$ satisfying

$$
\left\{x_{i}, y_{j}\right\}=\delta_{i j} .
$$

In the physical interpretation of this result we identify $x_{1}$ with the Hamiltonian. Then $y_{1}$ describes the time evolution of the system and the remaining elements form $(2 n-2)$ independent constants of the motion. Actually these are only defined locally and in general an attempt to extend their definition over the whole of phase space leads to their being multivalued $[23,24]$. Thus they are not constants of the motion in the ordinary sense and do not imply any degeneracy in the motion of the Classical system (Ref. [21], p. 288-299). On the other hand the analogous result in Quantum mechanics would lead to a degeneracy in the energy spectrum as long as we could show that the $x_{i}, y_{j}$ can be chosen to be elements of $\mathscr{L}(\mathscr{H})$ with $\mathscr{H}$ the representation space of the Hamiltonian. We stress that this latter condition should not be overlooked for otherwise there is no guarantee that all the unitary irreducible representations of the symmetry group will appear in the spectral decomposition of the Hamiltonian [25]. Apart from a postiori verification, this requirement can be checked by exhibiting the $x_{l}, y_{j}$ as polynomials in the canonical variables or as polynomials or weak limits of polynomials of their (bounded) exponentiated form. This fact has led to the conjecture [24] that the Quantum analogue of (11.3) can be solved for a given Hamiltonian (omitting the time) if and only if the Classical 
constants of the motion are defined globally, that is over the whole of phase space. However at the moment the evidence for this is limited to a few examples which include the harmonic oscillator and the hydrogen atom and we feel somewhat sceptical about its general validity. The above considerations lead to the following interesting problem $[9,10$, 23, 24, 26-29]. Given an arbitrary Lie algebra $\mathscr{L}$ find all possible expressions for the generators of $\mathscr{L}$, as elements of $A^{n}$ or $\mathscr{P}^{n}$. Now if $\mathscr{L}$ is finite dimensional we can always find at least one realisation as a bilinear function of the canonical operators $q_{i}, p_{j}: i, j=1,2 \ldots n$, for some $n$. This fact is an easy consequence of Ado's theorem (Ref. [13], p. 202). On the other hand there may also be other polynomials having this property. We have already noted two further examples in $\S 9$ for $s l(2, \mathbb{C})$. In these cases $n$ is equal to the rank of the Lie algebra. In general for semisimple Lie algebras it has been shown that $n$ must be at least equal to the rank $[10,28]$ though this condition is not sufficient for a realisation. Again in the Quantum case we should not always restrict ourselves to $\mathscr{P}$ for, as we saw in $\S 10$, interesting solutions may be so excluded.

The significance of canonical transformations to this problem arises because we should not want to distinguish canonically equivalent solutions. In this respect the Classical and Quantum brackets will behave differently and we can expect the latter to be more difficult to handle. In the case when one of the canonical variables appears at most linearly, then on account of the isomorphism implied by Corollary 6.1 the Classical and Quantum solutions become equivalent and both reduce to the problem of determining the so-called non-linear realisations of Lie algebras [30]. This can be handled by techniques developed in the theory of Lie groups [19]. However the wider problem described above has no such general formalism.

Acknowledgements. This work was developed mainly at Oxford whilst the author held a Junior Research Fellowship at Corpus Christi College and a Lectureship in the Mathematics department. It was completed at Tel-Aviv University under a Royal Society-Israel Academy Research Fellowship. My interest in the subject first came about through discussions with Dr. Wollenberg to whom much credit is due and to whom I should like to express my thanks. Some of the ideas developed here arose out of these conversations and from the work of Dr. Wollenberg referred to in the text. I should also like to thank Professor Coulson for his comments on the manuscript.

\section{References}

1. Souriau, J. M.: Commun. Math. Phys. 1, 374 (1966).

2. Streater, R. F.: Commun. Math. Phys. 2, 354 (1966).

3. Dirac, P. A. M.: Quantum mechanics, 4th Ed., Chap. IV, pp. 84-89. Oxford: Clarendon Press 1958. 
4. Abraham, R.: Foundations of mechanics, Chap. IV, pp. 132-153. New York: W. A. Benjamin 1967.

5. Shewell, J. R.: Am. J. Phys. 27, 16 (1959).

6. Groenewold, H. J.: Physica 12, 405 (1946).

7. Hermann, R.: Lie groups for physicists, Chap. 16, pp. 137-149. New York: W. A. Benjamin 1966.

8. Fradkin, D. M.: Progr. Theoret. Phys. 37, 798 (1967).

9. Mukunda, N.: Phys. Rev. 155, 1383 (1967).

10. Simoni, A., Zaccaria, F.: Nuovo Cimento 59 A, 280 (1969).

11. Pauli, W.: Handbuch der Physik, Book V, Part I, p. 50. Berlin-Göttingen-Heidelberg: Springer 1958.

12. Rosenbaum, D. M.: J. Math. Phys. 10, 1127 (1969).

13. Jacobson, N.: Lie algebras. New York: Interscience 1962.

14. Wollenberg, L. S.: Progress report no. 12, Wave mechanics group, Mathematical Institute, Oxford 1965-1966, p. 4.

15. - Proc. Am. Math. Soc. 20, 315 (1969).

16. Rosenbaum, D. M.: J. Math. Phys. 8, 1973 (1967).

17. Van Hove, L.: Acad. Roy. Belg. Bull. Classe Sci. Mém. (5) 37, 610 (1951).

18. Jauch, J. M.: Foundations of quantum mechanics, Chap. 12, pp. 195-205. London: Addison-Wesley Publ. Co. 1968.

19. Joseph, A., Solomon, A. I.: J. Math. Phys. 11, 748 (1970).

20. Kurşunoğlu, B.: Modern quantum theory, Chap. XIII, pp. 364-367. London: W. H. Freeman and Co. 1962.

21. Goldstein, J. W.: Classical mechanics, Chap. 8, pp. 255--258. London: AddisonWesley Publ. Co. 1962.

22. Eisenhart, L. P.: Continuous groups of transformations, Chap. VI, pp. $281-292$. New York: Dover Publ. 1961.

23. Maiella, G., Vitale, B.: Nuovo Cimento 57 A, 330 (1967).

24. Duimio, F., Pauri, M.: Nuovo Cimento 51 A, 1141 (1967).

25. Cisneros, A., McIntosh, H. V.: J. Math. Phys. 10, 277 (1969).

26. Pauri, M., Prosperi, G.: J. Math. Phys. 7, 366 (1966).

27. Simoni, A., Zaccaria, F., Vitale, B.: Nuovo Cimento 51 A, 448 (1967).

28. Guest, P. B.: Nuovo Cimento 61 A, 593 (1969).

29. Chow, Y.: J. Math. Phys. 10, 975 (1969).

30. Coleman, S., Wess, J., Zumino, B.: Phys. Rev. 177, 2239 (1969).

A. Joseph

Physics Department

Tel-Aviv University

Tel-Aviv, Israel 